\title{
Comparison of Analytical Methodologies for Aluminium Determination in Hemodialysis Fluids
}

\author{
Miriam B. Quirós* \\ Centro de Electroquímica y Energía Química, Universidad de Costa Rica, Apdo 2060, \\ San Pedro, Ciudad Universitaria Rodrigo Facio, Costa Rica
}

\begin{abstract}
In order to quantifying aluminium by analyzing hemodialysis fluids, two methodologies were developed and validated: square wave adsorptive voltammetry (SWAV) and electrothermic absortion. Performance parameters were established for the two methodologies. They were both applied successfully to the analysis of hemodialysis fluids.
\end{abstract}

Keywords: aluminium, analysis, hemodialysis, fluids, square wave voltammetry, adsorptive electrothermic absorption, performance parameters.

\section{Introducción}

El aluminio desde hace muchos años se ha considerado un problema real en los fluidos empleados en hemodiálisis renal, su uso continuado causa acumulación en los pacientes expuestos aún a bajas concentraciones, se ha considerado un contaminante iatrogénico y está presente en el ambiente.

La toxicidad del aluminio en diálisis renal es un hecho conocido [1]; el aluminio por muchos años ha sido señalado como causante de daños óseos y neurológicos: encefalopatías y enfermedades óseas como la enfermedad adinámica del hueso asociada a baja reconversión y a bajos contenidos de Al, la osteítis fibrosa asociada a la alta reconversión, hipertiroidismo y altos contenidos de Al [2].

Durante más de 30 años se ha determinado por diversas metodologías como la absorción atómica con horno de grafito [3]' y combinada con resinas de amberlita modificadas con agentes quelantes para preconcentrar el aluminio [4], la detección espectrofotométrica con preconcentración de flujo [5] y voltamperometrías adsortivas [6,7], las cuales son muy sensibles. Los

\footnotetext{
* Corresponding author. E-mail address: miriamdemorales@yahoo.com
} 
procedimientos empleados deben ser confiables, por lo que se hace necesario contar con metodologías analíticas validadas para la cuantificación de bajas concentraciones del aluminio presente en las disoluciones empleadas en las máquinas dializadoras.

El aluminio es difícil de determinar a bajas concentraciones debido a que: en absorción atómica con horno de grafito, existe la necesidad de preconcentrar o utilizar modificadores de matriz [8,9] y esto produce contaminación que cambia la concentración de la muestra. Los fluidos de hemodiálisis se han analizado por técnicas polarográficas [10]. Las técnicas voltametrias adsortivas son lentas y exigen una regulación cuidadosa del $\mathrm{pH}$. La contaminación con aluminio externo es difícil de evitar y requiere limpieza óptima de la cristalería empleada. La muestra se debe tratar para maximizar la recuperación del analito. Existen diferentes fuentes de contaminación con aluminio como el aluminio del agua utilizada para preparar fluidos de diálisis, antiácidos o geles de aluminio, enlazantes de fosfatos, floculantes de aluminio empleados en tratamiento de aguas para uso doméstico y aluminio de utensilios o envolturas en contacto con alimentos. Debido a estas fuentes de contaminación la legislación internacional sobre diálisis y dado que un paciente de hemodiálisis está expuesto a 2000030000 litros/año, ha publicado las siguientes normas en relación con la calidad del agua y los sistemas de dializado, que se refieren a la pureza química y pureza microbiológica: Norma Europea Section IV. Dialysis fluid purity [11], American Association Advancement Medical Instrumemtation (AAMI [12]) y la Norma Australiana [13]. Los sistemas existentes para tratamiento del agua utilizada en diálisis como filtros de carbón, sistemas de intercambio iónico, sistemas de electrodiálisis, o los que emplean ósmosis inversa: nanofiltración, ultrafiltración, no siempre proporcionan la eliminación requerida de los iones presentes. En Costa Rica se realiza hemodiálisis en hospitales con máquinas dializadoras y se realiza diálisis peritoneal ambulatoria en casas con soluciones parenterales $\mathrm{o}$ automatizada [2].

\section{Parte experimental del complejo aluminio con cupferron}

Se utilizó un polarógrafo PARC modelo 384 B acoplado a un sistema de tres electrodos $303 \mathrm{~A}$, constituido por un electrodo de trabajo de gota suspendida de mercurio, un electrodo de plata / cloruro de plata como referencia, y un electrodo de platino como indicador. El software empleado fue desarrollado específicamente para controlar el equipo utilizado [14].

El material volumétrico empleado se colocó por una semana en un baño de $\mathrm{HNO}_{3}$ al $20 \%$ v/v y luego se enjuagó con agua Milli Q y se reflujó por una hora con $\mathrm{HCl}: \mathrm{HNO}_{3}$; en la proporción 3:1, ambos destilados sobre cuarzo, se enjuagó con agua Milli Q destilada posteriormente, se secó cubierto y se guardó en doble bolsa plástica.

Se utilizó un buffer de NaOAc 2 mol/L ajustado a pH 7 con $\mathrm{NaOH}$, ambos de calidad Suprapur, el cupferrón 0,060 mol/L a partir del reactivo de calidad para análisis, se preparó diariamente con agua Milli $\mathrm{Q}$, desoxigenada con $\mathrm{N}_{2}$, y con $\mathrm{NH}_{3}$ Suprapuro al 2\% v/v. Se utilizó un Tritrisol de aluminio. 
La parte experimental se realizó en tres etapas: optimización de las condiciones experimentales de medición, validación del método desarrollado y determinación del proceso de digestión adecuado para los fluidos de hemodiálisis. Se seleccionó la técnica de SWV porque la señal era definida y mayor que para la misma concentración en DPS. La optimización secuencial de los parámetros para SWV se realizó para el potencial de acumulación, tiempo de acumulación, frecuencia, amplitud de pulso, tiempo de equilibrio, concentración de cupferrón, pH óptimo, pero la determinación del pH adecuado se realizó antes de optimizar los parámetros de medición experimentales.

Los valores optimizados de la técnica (SWV), para la determinación de aluminio fueron: potencial de acumulación $(-0,4 \mathrm{~V})$, tiempo de acumulación (60 s), altura de pulso $(50 \mathrm{mV})$, frecuencia $(25 \mathrm{~Hz})$, tiempo de equilibrio $(20 \mathrm{~s})$, concentración de cupferrón $\left(2 \times 10^{-4} \mathrm{~mol} / \mathrm{L}\right), \mathrm{pH}, \quad(7,00)$. Las condiciones optimizadas instrumentales de medición fueron: potencial inicial $(-0,4 \mathrm{~V}$ vs. $\mathrm{Ag} / \mathrm{AgCl})$, potencial final, $(-1,4 \mathrm{~V}$ vs. $(\mathrm{Ag} / \mathrm{AgCl})$, potencial de acondicionamiento, $(0 \mathrm{~V}$ vs. $\mathrm{Ag} / \mathrm{AgCl})$, amplitud de pulso $(0,020 \mathrm{~V})$, tiempo de desoxigenación inicial (200 $\mathrm{s})$, tiempo de desoxigenación entre réplicas (20 s), tiempo de preconcentración (60 s), tiempo de acondicionamiento (0 s), tiempo de equilibrio (20 s), incremento de barrido $(2 \mathrm{mV})$, frecuencia $(25 \mathrm{~Hz})$, ciclos (1).

\section{Parte experimental de la determinación electrotérmica de aluminio}

Las muestras se colectaron durante un año en la unidad de hemodiálisis del hospital México de la CCSS, en frascos de Nalgeno lavados con EDTA al 5\% $\mathrm{m} / \mathrm{v}$, enjuagados con agua de Milli Q destilada; posteriormente, se acidificaron, se congelaron a $-30{ }^{\circ} \mathrm{C}$ hasta su análisis. La cristalería se trató del mismo modo en ambas determinaciones. Se tomaron alícuotas de $12,50 \mathrm{~mL}$ de las muestras y se diluyeron a $25,00 \mathrm{~mL}$, con $\mathrm{HNO}_{3}$ destilado $0,5 \% \mathrm{~m} / \mathrm{v}$, se midieron en un Varian Modelo Spectra AA 220 equipado con automuestrador a 396,2 nm, las alícuotas de muestra inyectadas fueron de $10 \mu \mathrm{L}$. Se evaluó la veracidad en cada corrida con adiciones triples de $12 \mu \mathrm{g} / \mathrm{L}, 24 \mu \mathrm{g} / \mathrm{L}, 48 \mu \mathrm{g} / \mathrm{L}$ a tres réplicas de muestra.

\section{Resultados de la determinación voltamperométrica de aluminio con cupferrón}

\section{Validación del método desarrollado}

El método desarrollado se validó a través de la determinación de sus límites de detección y cuantificación, ámbito lineal, comprobación de la veracidad del método.

Se determinaron de la Fig. 1 los límites de detección y cuantificación a partir de la desviación estándar de las curvas de calibración [15].

En la Fig. 2 se determinó la linealidad de las curvas de calibración hasta $60 \mu \mathrm{g} / \mathrm{L}$ de aluminio adicionados en celda; este ámbito de concentración se consideró adecuado para las concentraciones de aluminio en los fluidos empleados en hemodiálisis. 


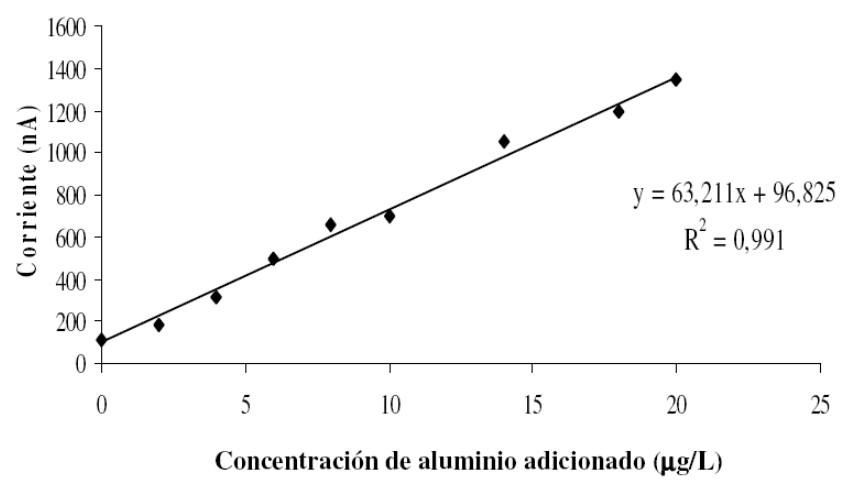

Figura 1. Recta de calibración para determinación del límite de detección para Al por voltametría adsortiva.

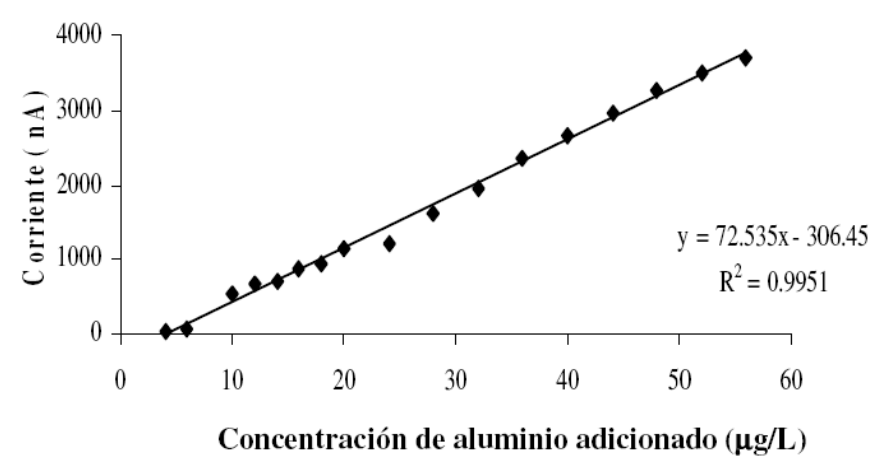

Figura 2. Recta de calibración para determinación del ámbito lineal para Al por voltametría adsortiva.

\section{Determinación de la veracidad del método}

Se mineralizaron con $\mathrm{HNO}_{3} 12$ disoluciones acuosas, a las cuales se le adicionaron $0 \mu \mathrm{g} / \mathrm{L}, 4 \mu \mathrm{g} / \mathrm{L}$ y $20 \mu \mathrm{g} / \mathrm{L}$ de aluminio y se analizaron por el método desarrollado, se restó el promedio de los blancos al promedio de muestras, la recuperación obtenida para $4 \mu \mathrm{g} / \mathrm{L}$ fue $150 \%$, la recuperación obtenida para 20 $\mu \mathrm{g} / \mathrm{L}$ fue $98,2 \%$. Los parámetros de desempeño se muestran en Cuadro 1. El método optimizado y validado se aplicó a la determinación de muestras de fluidos de hemodiálisis.

Cuadro 1. Concentración de aluminio $(\mu \mathrm{g} / \mathrm{L})$ en fluidos de hemodiálisis analizados por absorción electrotérmica.

\begin{tabular}{|c|c|c|c|c|c|c|c|c|c|c|c|c|c|}
\hline & Junio & Agosto & Setiem & Octubre & Noviem & Diciem & Enero & Febrero & Marzo & Abril & Mayo & Junio & Julio \\
\hline M1 & 27 & 29 & 15 & 14 & & & & & & 30 & 4 & 4 & \\
\hline M2 & 48 & 31 & 19 & 9 & 24 & 86 & 26 & ND & 71 & $\mathrm{D}$ & & $\mathrm{D}$ & 8 \\
\hline M3 & 10 & 8 & 18 & 31 & 23 & 22 & 22 & 8 & ND & 19 & 24 & 28 & 33 \\
\hline M4 & 13 & 8 & 28 & 27 & 16 & 23 & 201 & ND & ND & 12 & 11 & 17 & 54 \\
\hline M5 & 27 & 0 & 36 & 16 & 26 & 27 & 25 & ND & ND & 6 & 8 & 7 & 22 \\
\hline M6 & 10 & 8 & 20 & 13 & 20 & $\mathrm{D}$ & 46 & ND & ND & 8 & 14 & & 13 \\
\hline M7 & 22 & 11 & 18 & 18 & 15 & 14 & 17 & ND & ND & 11 & & 3 & \\
\hline M8 & 12 & ND & 27 & 20 & 18 & 33 & 52 & ND & ND & 15 & 10 & 11 & 8 \\
\hline M9 & 13 & ND & 35 & 16 & 46 & 17 & 17 & ND & ND & 11 & 33 & 6 & \\
\hline M10 & 14 & ND & 23 & 7 & 7 & 20 & 18 & ND & ND & 9 & 20 & $\mathrm{D}$ & 4 \\
\hline $\mathrm{C4}$ & 17 & 16 & 20 & & 106 & 12 & 39 & & ND & & & 12 & 7 \\
\hline
\end{tabular}




\section{Análisis de muestras por voltamperometría}

El análisis de muestras de fluidos empleados en la hemodiálisis se realizó previo lavado del material de Nalgeno con $\mathrm{HNO}_{3}$ al $20 \%$ v/v, se adicionaron $100 \mu \mathrm{L}$ de $\mathrm{HNO}_{3}$ de calidad S.P al recipiente antes de tomar la muestra. Ésta estaba diluida en las máquinas dializadoras, a la concentración apropiada para el tratamiento de los pacientes. Se empacaron en doble bolsa plástica y se rotularon. Se transportaron rápidamente hasta el laboratorio, se almacenaron en congelación a $-30{ }^{\circ} \mathrm{C}$. Se midieron $4,00 \mathrm{~mL}$ de muestra y se colocaron en material descontaminado: los balones de $25,00 \mathrm{~mL}$ se trataron con $\mathrm{HCl}$ y $\mathrm{HNO}_{3}$, en la proporción 3/1, se calentaron a ebullición y se lavaron con agua Milli-Q. Se mineralizaron las muestras, se aforaron, se midió una masa de muestra y se colocó en la celda polarográfica, se reguló el pH de la muestra a 7,0 con ayuda de un pHmetro y disoluciones de HOAC, NaOAC, 2 mol/L y NaOH 1 mol/L, según fuera necesario. El control del $\mathrm{pH}$ para las muestras fue muy estricto para formar el complejo. Se adicionó una alícuota de $40 \mu \mathrm{L}$ de cupferron, se ajustó con agua MilliQ a una masa final de $10,00 \mathrm{~g}$ y se cuantificó por adición estándar. El análisis de las muestras se realizó de manera aleatoria, se muestran en el Cuadro 2.

Cuadro 2. Concentración de aluminio en fluidos de hemodiálisis por voltamperometría adsortiva SWV.

\begin{tabular}{|l|c|c|c|}
\hline Muestra & Concentración $\boldsymbol{\mu g} / \mathbf{L}$ & Muestra & Concentración $\mathbf{\mu g} / \mathbf{L}$ \\
\hline $\mathbf{1 3}$ & & $\mathbf{1}$ & \\
\hline Réplica 1 & D & Réplica 1 & 74,6 \\
\hline Réplica 2 & D & $\mathbf{2}$ & \\
\hline $\mathbf{5}$ & & Réplica 1 & 72,02 \\
\hline Réplica 1 & 14,9 & $\mathbf{9}$ & \\
\hline Réplica 2 & 10,9 & Réplica 1 & 322 \\
\hline $\mathbf{4}$ & & Réplica 2 & 392 \\
\hline Réplica 1 & 18,9 & $\mathbf{8}$ & \\
\hline $\mathbf{1 2}$ & & Réplica 1 & 34.7 \\
\hline Réplica 1 & N.D & $\mathbf{6}$ & \\
\hline Réplica 2 & N.D & Réplica 1 & 22,0 \\
\hline $\mathbf{1 1}$ & & Réplica 2 & 24,0 \\
\hline Réplica 1 & 25,6 & $\mathbf{3}$ & \\
\hline Réplica 2 & 40,4 & Réplica 1 & 44,6 \\
\hline & & Réplica 2 & 13,4 \\
\hline
\end{tabular}

\section{Resultados de la determinación de aluminio por absorción electrotérmica Figuras de mérito del método electrotérmico}

Los limites de detección y cuantificación (LD y LC respectivamente) se determinaron de las curvas de calibración de acuerdo con Miller y Millar [15].

La veracidad se determinó por adición de cantidades conocidas de aluminio a muestras, se restó la concentración de la muestra y se calculó el C.V. del aluminio recuperado. El ámbito lineal se determinó por la medida de patrones de aluminio, se muestra en la Fig. 3 y de la Fig. 4, se observa que cerca de $100 \mu \mathrm{g} / \mathrm{L}$ 
el coeficiente de correlación disminuye. La exactitud se evalúo por análisis de siete réplicas de una muestra certificada de agua; CMR- TMW HPS Certified Reference Material Trace Metals in Drinking Water, Lot \# 005914 que contenía $120 \mu \mathrm{g} / \mathrm{L}$ de aluminio: se tomaron para cada réplica 2,00 $\mathrm{mL}$ de la muestra certificada, se acidificaron al $0,5 \% \mathrm{v} / \mathrm{v}$ con $\mathrm{HNO}_{3}$ destilado, se diluyeron a un volumen de 10,00 mL. Se interpoló en una curva de calibración. En el Cuadro 3 se muestra el desempeño de los métodos validados.

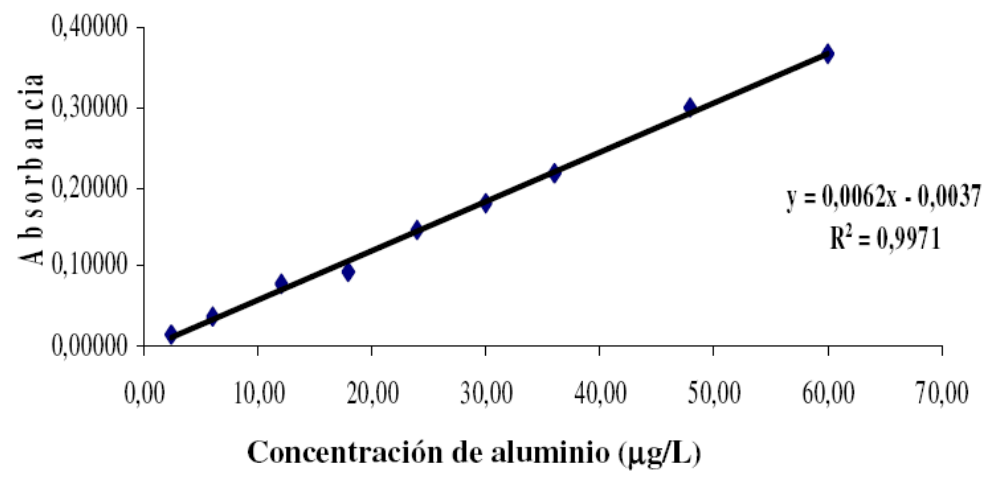

Figura 3. Recta de calibración para la determinación de Al en fluidos de hemodiálisis por absorción electrotérmica.

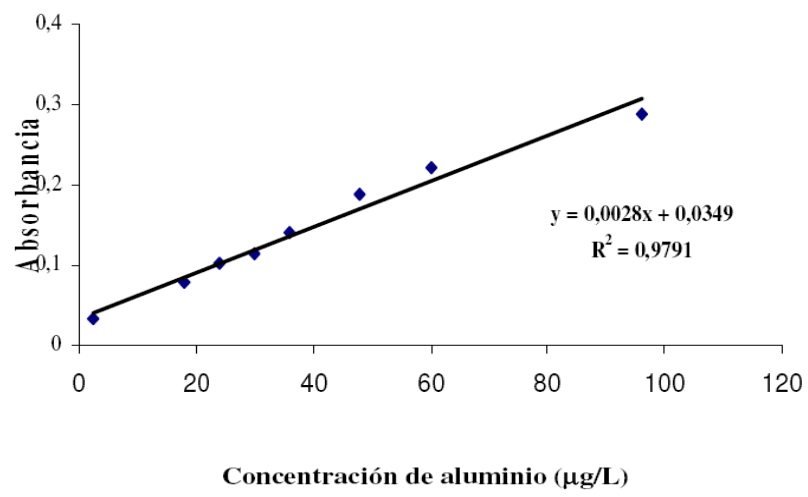

Figura 4. Recta de calibración para la determinación del ámbito lineal para aluminio en fluidos de hemodiálisis por absorción electrotérmica.

Cuadro 3. Comparación de los parámetros de desempeño de ambos métodos.

\begin{tabular}{|l|c|c|}
\hline & Voltamperometría & Absorción electrotérmica \\
\hline Límite de detección & $2 \mu \mathrm{g} / \mathrm{L}$ & $1 \mu \mathrm{g} / \mathrm{L}$ \\
\hline Límite de cuantificación & $7 \mu \mathrm{g} / \mathrm{L}$ & $3 \mu \mathrm{g} / \mathrm{L}$ \\
\hline Ámbito lineal & $60 \mu \mathrm{g} / \mathrm{L}$ & $2,4-96 \mu \mathrm{g} / \mathrm{L}$ \\
\hline Veracidad 20 $\boldsymbol{\mu g} / \mathbf{L} \mathbf{~ n = 7}$ & $98 \%$ & $113 \%(12 \mu \mathrm{g} / \mathrm{l}, \mathrm{n}=3)$ \\
Veracidad 4 $\boldsymbol{\mu g} / \mathbf{L} \mathbf{~ n = 7}$ & $150 \%$ & $81 \%(24 \mu \mathrm{g} / \mathrm{l}, \mathrm{n}=3)$ \\
& & $99 \%(48 \mu \mathrm{g} / \mathrm{l}, \mathrm{n}=3)$ \\
\hline Coeficiente de variación en muestras & $17 \%$ & $25 \%(4 \mu \mathrm{g} / \mathrm{L}, \mathrm{n}=3)$ \\
& & $10 \%(23 \mu \mathrm{g} / \mathrm{L}, \mathrm{n}=3)$ \\
\hline Exactitud & No se determinó & $112 \pm 10 \%(\mathrm{n}=7)$ \\
\hline
\end{tabular}




\section{Análisis de muestras de fluidos de hemodiálisis por absorción electrotérmica}

Las muestras se colectaron en frascos de Nalgeno lavado con EDTA al $5 \% \mathrm{~m} / \mathrm{v}$, enjuagado con agua de Milli Q destilada posteriormente, se acidificaron, se congelaron a $-30{ }^{\circ} \mathrm{C}$ hasta su análisis. Se tomaron alícuotas de $12,50 \mathrm{~mL}$ de las muestras y se diluyeron a $25,00 \mathrm{~mL}$, con $\mathrm{HNO}_{3}$ destilado $0,5 \% \mathrm{~m} / \mathrm{v}$, se midieron en un Varian Modelo Spectra AA 220 equipado con automuestrador Varian PSD 110, y un horno de grafito GTA 110 a la longitud de onda de 396,2 nm. Las alícuotas de muestra inyectadas fueron de $10 \mu \mathrm{L}$ y se colocaron en recipientes de Nalgeno para el automuestrador con volumen de $2 \mathrm{~mL}$. Se evaluó la veracidad en cada corrida con adiciones triples de $12 \mu \mathrm{g} / \mathrm{L}, 24 \mu \mathrm{g} / \mathrm{L}, 48 \mu \mathrm{g} / \mathrm{L}$ a tres réplicas de muestra. Se muestran resultados de las muestras analizadas en Cuadro 1.

\section{Discusión y conclusiones}

Ambos métodos validados se pueden usar para la determinación de aluminio en fluidos de hemodiálisis y tienen límites de detección comparables. Los coeficientes de variación son apropiados para las concentraciones analizadas. Las muestras analizadas por ambos métodos provenían de las mismas máquinas dializadoras, pero se analizaron en períodos diferentes de tiempo, por lo que los resultados no son comparables.

Lo destacable es la posibilidad de poder emplear estos métodos como confirmatorios de forma recíproca en casos donde sea necesario. Los procedimientos de limpieza de recipientes y cristalería son críticos para que la muestra no se contamine. La concentración de aluminio en los fluidos de hemodiálisis es muy baja por lo que los métodos deben ser muy sensibles.

El horno de grafito usando automuestrador, permite realizar muchas más determinaciones que la voltamperometría adsortiva, siendo esta técnica apropiada para confirmación y el horno de grafito para el control de calidad.

\section{Referencias}

1. R. Vanholder et al., The role of trace elements in uraemic toxicity, Nephrol Dial Transplant 17 [ Suppl 2] (2002) 2-8.

2. M. Barquero Quirós, R. Vargas Rojas, R Blanco-Sáenz, Neurotoxicidad y enfermedades óseas provocadas por la contaminación con aluminio en las soluciones de diálisis renal. Rev. Costarricense de Ciencias Médicas $22(3,4)$ (2001) 177-189.

3. J. Smeyers-Verbeke, D. Verbeelen, Determination of aluminiun in dializate concentrates by L'vov platform graphite furnace atomic absortion spectrometry, Anal. Chem. 60 (1988) 380-383.

4. I. Narim, M. Tuzen, M Soylac, Aluminium determination in enviromental samples by graphite furnace atomic absortion spectrometry after solid phase extraction on amberlite XAD- 1180/ pyrocathecol violet chelating resin, Talanta 63 (2004) 411418.

5. M. do S. Silva, P.B. Freire dos Reis, Determinação espectrofométrica de alumínio em concentrados salinos utilizados en hemodiálise empregando pré-concentração em fluxo, Quim. Nova 25-6 (2002) 931-934. 
6. M. Barquero Quirós, Biyun Z. Wu, Development and validation of an adsortive method for aluminiun determination in fluids used in renal dialysis. Portugaliae Electrochimica Acta 23 (2005) 165-178.

7. M. Barquero Quirós, Desarrollo y optimización de un método voltamperométrico adsortivo para la determinación de aluminio en agua, Portugaliae Electrochimica Acta 23 (2005) 403-411.

8. W. Slavin, G.R. Carnrick, D.C. Manning, Nitrate magnesium as a matrix modifier in the stabilized temperature platform furnace, Anal. Chem. 54 (1982) 621-624.

9. W. Slavin, G.R. Carnrick, D.C. Manning, Effect of graphite furnace substrate materials on analysis by furnace graphite atomic absortion, Anal. Chem. 53 (1981) 1504-1509.

10. A. Romero, J. Tahán, A. Moronta, Alternative Simple Mineralization Procedures to Permit Subsequent Polarographic Determination of Total Soluble Aluminiun in Haemodialysis Water, Analytica Chimica Acta 257 (1992) 147.

11. Guidelines. Section IV. Dialysis fluid Purity. Nephrol. Dial. Transplant 17 [Suppl 7] (2002) 45-62.

12. Association for Advancement of Medical Inst., Arlington, VA, AAMI American National Standard, (1995) pp. 257-277.

13. P. Kerr, V. Perkovic, J. Petrie, J. Agar, A. Disney, Nephrology. Dialysis Adequacy (HD) Guidelines. 10 (2005) S61-S80.

14. León R. Carlos, Tesis de Doctorado 2002, Universidad de Extremadura, España.

15. James N. Miller, Jane C. Miller, Estadistica y Quimiometría para Química Analítica. $4^{\mathrm{a}}$ Edición, Prentice Hall, 2002. Capítulo 5.

\section{Comparación de Metodologías Analíticas para Determinación de Aluminio en Fluidos de Hemodiálisis}

\section{Resumen}

Se desarrolló y validó una metodología para cuantificar aluminio en fluidos utilizados en hemodiálisis renal, basada en la reacción de Al con cupferrón, adsorción del complejo sobre un electrodo de gota de $\mathrm{Hg}$ y la posterior desorción y medida de la corriente producida por la reducción del complejo. Se realizó la validación de parámetros para voltamperometría de onda cuadrada (SWV). Se determinaron también los parámetros de desempeño del método, veracidad, ámbito lineal, límite de detección, límite de cuantificación. Se aplicó a la determinación de aluminio en muestras de hemodiálisis.

Se desarrolló y validó posteriormente una metodología para la determinación de aluminio en fluidos empleados en hemodiálisis renal por absorción electrotérmica. Se determinaron las mejores condiciones para el tratamiento de la muestra. También se determinaron los parámetros de desempeño del método. Esta metodología se aplicó durante un año al análisis de los fluidos de la Unidad de Hemodiálisis del Hospital México de la Caja Costarricense del Seguro Social de Costa Rica. El contenido en $\mu \mathrm{g} / \mathrm{L}$ de la mayor parte de las muestras analizadas por ambas metodologías se encontraban sobre las recomendaciones internacionales en agua empleada en hemodiálisis.

Palabras clave: aluminio, hemodiálisis, determinación, voltametría de onda cuadrada, adsorción, validación, absorción electrotérmica. 